\title{
I ruoli del formatore tra tradizione e ricerca di una nuova identità
}

\section{Luca Bausch}

La modularizzazione dei percorsi costituisce una risposta alla crescente domanda di flessibilizzazione e individualizzazione della formazione. Se da un lato questi processi sono forieri di un potenziale di emancipazione considerevole, dall'altro possono generare insicurezza e dipendenza, tali da rendere auspicabile l'introduzione di misure atte a ricomporre l'eterogeneità di percorsi composti da unità più o meno indipendenti e quindi portatrici di logiche interne di volta in volta diverse. Dalle nostre riflessioni - che, a partire dalle esperienze condotte presso l'ISPFP, si incentrano sulle funzioni che i professionisti della formazione sono chiamati ad assumere in questi nuovi contesti - sembrano emergere tre ruoli di formatore: il manager, il docente $e$ l'accompagnatore. Se al primo competono principalmente compiti legati alla struttura dei percorsi e alla loro gestione, al docente - la cui attività si esplica normalmente all'interno del quadro ben definito di un modulo - spetta di ristrutturare la conoscenza (tendenzialmente privata del suo referente naturale, la disciplina) secondo nuovi criteri d'ordine, ad esempio il profilo di competenza mirato. Sempre maggior rilevanza assumono le funzioni legate all'accompagnamento che trovano il loro terreno di applicazione negli aspetti relazionali (punti di riferimento per la persona in formazione) e nel supporto ai processi di apprendimento (metacognizione, impiego di strumenti di formazione diversificati). L'azione formativa tende dunque a concentrarsi sui suoi aspetti metodologici e relazionali con una particolare attenzione ai processi di attribuzione di senso che, nel contesto di strutture modulari, non possono piu essere dati per scontati.

Le funzioni che caratterizzano i tre profili emersi possono combinarsi in maniera diversa in rapporto alle situazioni contingenti e in particolare alla tipologia di percorso modulare. Questo ci pone di fronte ad una serie di interrogativi relativi ai processi di costruzione dell'identità professionale: se la tendenza emergente è quella di una suddivisione del lavoro che vede le tre figure sempre piu specializzate nei rispettivi settori di competenza, quali sono le rappresentazioni e attese indotte nelle persone in formazione? Quale la percezione, in termini di identità, che il formatore può avere di se stesso in quanto professionista? 


\section{Premessa}

Chi si occupa di formazione professionale non può prescindere da un'attenta analisi degli effetti che i mutamenti in atto a tutti i livelli nel mondo della formazione hanno su quegli attori che recitano pur sempre uno dei ruoli chiave nei processi di apprendimento: i formatori. Non si può infatti pensare che le professioni di docente, insegnante o formatore a diversi livelli restino impermeabili all'evoluzione che interessa il contesto nel quale operano, quello delle istituzione formative in primo luogo.

Il ritmo dell'evoluzione chiede a chi si occupa di formazione di adeguare le proprie strutture; in certa misura più che di un mero adattamento si dovrebbe parlare di «anticipazione» nel senso che le istituzioni preposte alla formazione - professionale in particolare - dovrebbero potersi dotare di strumenti in grado di cogliere i mutamenti in atto e prevederne la direzione. Anche la formazione deve fare proprie quelle caratteristiche di versatilità e di flessibilità che sembrano essere divenute le condizioni imprescindibili per agire nel mondo economico e sociale contemporaneo.

Tra le risposte a queste domande di flessibilità e di adattamento che incontrano il favore delle istanze formative trova un posto di rilievo l'offerta di percorsi di formazione strutturati in moduli, ovvero in unità più o meno autonome.

Strutturare e quindi delimitare la conoscenza in sequenze finite significa mutare l'approccio alla conoscenza stessa. Alla costruzione progressiva e soggetta a logiche lineari si sostituisce un approccio reticolare dove le relazioni tra i nodi della rete (i moduli) non sono evidenti o comunque non predeterminati dalla struttura. In linea di principio ogni modulo è autosufficiente e i percorsi suscettibili di adattarsi ad esigenze di volta in volta diverse, pena il venir meno di quella flessibilità e individualizzazione di cui la modularità si fa promotrice. Ci bastino queste poche considerazioni per far emergere l'ambivalenza che caratterizza l'approccio modulare: se da un lato si rivela portatore di un importante potenziale di emancipazione, dall'altro può generare nuove situazioni di dipendenza e insicurezza. Si tratta in fondo di una particolare declinazione di quella stessa ambivalenza che sembra soggiacere alle dinamiche che governano il mondo contemporaneo.

Il settore della formazione professionale appare sollecitato in maniera più marcata dalle esigenze sopra accennate in quanto particolarmente sensibile all'evoluzione economica e sociale. I rapidi mutamenti nella definizione delle competenze necessarie allo svolgimento di una determinata professione e l'affacciarsi di nuovi profili professionali, richiedono una profonda riflessione sulle modalità $\mathrm{e}$ sul senso stesso dell'apprendimento.

I confini tra attività professionale e formazione si vanno ridefinendo sia in termini istituzionali che cronologici: da un lato scuola e luogo di lavoro non sono più così nettamente distinguibili, dall'altro le biografie professionali non 
presentano più la netta distinzione tra et à dell'apprendimento e vita professionale intersecandosi i due momenti durante tutto l'arco della vita. Questa ridefinizione interessa $\mathrm{i}$ confini tra apprendimento e attività professionale che si vanno assottigliando anche da un punto di vista concettuale: In che modo si apprende? Con quali strumenti? In quali situazioni? Su quali contenuti?

Se pensiamo ad esempio all'interesse che hanno suscitato negli ultimi 2 decenni gli studi sull'apprendimento informale (ad es. Suchman, 1987, Hutchins, 1994), alle teorie che fanno dell'azione in situazione ${ }^{1}$ il loro terreno privilegiato di ricerca o agli approcci che si richiamano più o meno direttamente all'intuizione di Schön (1983) sulla pratica riflessiva, appare evidente come il limite tra attività produttiva e apprendimento sia sempre più labile.

La definizione stessa del sapere è rimessa in discussione: si pensi al concetto di competenza $^{2}$ e agli influssi che questo ha sul ruolo della conoscenza - posta sempre più in relazione con capacità e atteggiamenti -, sulla definizione dei profili professionali o ancora sul rapporto tra acquisizione e esercizio delle competenze stesse: la misura della professionalità non è più costituita dalle conoscenze ma dalla capacità di agire in situazione o, se si preferisce, dall'attitudine a mobilitare risorse in situazione.

Come si inserisce in questo contesto il formatore? Quali ruoli è chiamato ad assumere? Come si va delineando il suo (i suoi?) profilo professionale? Come sono vissuti dai formatori stessi, in termini di percezione di identità, i mutamenti in atto?

Se il tema del ruolo del formatore è tradizionalmente importante nella ricerca in ambito scolastico, lo sta diventando anche nel contesto professionale, a cominciare dalla letteratura specificatamente dedicata alla formazione in azienda. $\mathrm{Ci}$ sembra per contro che poca attenzione sia accordata ad un elemento importante, ovvero la dimensione soggettiva del formatore (Schürch, 2001): egli stesso è attore dei processi di apprendimento nella misura in cui li interpreta e li carica di significato.

Il presente contributo prende spunto dalle riflessioni promosse dell'Istituto Svizzero di Pedagogia per la Formazione Professionale di Lugano [ISPFP] ${ }^{3}$ nell'ambito della formazione professionale; in particolare faremo riferimento alle seguenti esperienze di formazione modulare portate a termine o in corso di svolgimento:

- Abilitazione per i docenti delle scuole professionali: si tratta della formazione pedagogica e didattica degli insegnanti della scuola professionale, organizzata in buona parte su base modulare. Annualmente vengono formati ca. 30 insegnanti in collaborazione con l'Università della Svizzera Italiana (Istituto svizzero di pedagogica per la formazione professionale) e l'Alta Scuola Pedagogica (Locarno);

- Formazione dei Formatori Aziendali (FFA): si articola in corsi strutturati in più livelli tematici, dalle caratteristiche dell'apprendimento dell'adulto alla 
progettazione e alla strutturazione didattica di corsi. Negli ultimi anni la frequenza media nei vari livelli è stata di una cinquantina di persone;

- Web Project Manager (WPM): sono stati formati a questa nuova figura professionale, riconosciuta dall'Ufficio Federale della Formazione e della Tecnologia, una trentina di professionisti in due corsi realizzati in collaborazione con diverse università svizzere e italiane;

- Assistenti di Pratica in Comunicazione mediata dal Computer (AP-CmC): si tratta di una formazione specifica per assistenti alla formazione e/o alla gestione di progetti che fanno ricorso alle tecnologie della comunicazione. Sono state formate una quindicina di persone;

- Referente in Pari Opportunità (RPO): per questa nuova professione sono stati formati 19 persone attive nella promozione delle pari opportunità tra i sessi, in particolare sui luoghi di lavoro ${ }^{4}$.

In rapporto all'evoluzione del ruolo dei formatori, abbiamo integrato le nostre riflessioni con quanto scaturito da una ricerca condotta negli anni $1999-2000$ sul terreno del Progetto Poschiavo ${ }^{5}$ sostenuta dal programma prioritario del FNR 6 Domani la Svizzera. Pur non occupandosi direttamente di modularità, la ricerca fornisce indicazioni interessanti sui mutamenti in termini di competenze necessarie all'azione sul terreno di una figura di formatore emergente: l'accompagnatore di percorsi di formazione.

Non è quindi il nostro un contributo emerso da una ricerca specifica quanto piuttosto il frutto di una serie di riflessioni a posteriori originate dalla ricerca e dall'analisi di esperienze di terreno.

\section{I significati della formazione.}

Una delle parole d'ordine che l'evoluzione del mondo del lavoro ha portato in auge è senza dubbio flessibilità. Flessibilità che può coniugarsi in più modi, dalla disponibilità a trasferirsi fisicamente per lavorare (in relazione allo spazio), a quella di lavorare in fasce orarie variabili (flessibilità dei tempi) fino all'impiego precario. Più vicino al nostro campo di riflessione troviamo la disponibilità richiesta alla forza lavoro di sapersi adattare ai continui mutamenti, a ridefinire il proprio profilo professionale, a prendere in mano la propria carriera professionale costruendosi un profilo di competenze versatile e quindi spendibile in più ambiti professionali; i concetti di long life learning e di employability fanno stato di questa discussione (Camus, 2003). Se da un lato le dinamiche emergenti del mondo del lavoro contribuiscono ad aumentare le possibilità offerte garantendo una maggiore mobilità e la possibilità/esigenza di adeguare la propria posizione professionale, il rovescio della medaglia è costituito da fattori quali il rischio e l'incertezza, forieri di frustrazioni per chi viene escluso da queste dinamiche. Come fa notare Sennett (1999), le conseguenze possono manifestarsi in un senso di fallimento di chi non riesce - o crede di non riuscire - a rispondere adeguata- 
mente alle nuove attese, nella rottura della continuità dell'esistenza e, di conseguenza, nell'erosione dell'integrità dell'io.

Il diritto di ritagliarsi un proprio ruolo in processi evolutivi sempre più rapidi può facilmente tramutarsi nell'ingiunzione a prendersi in mano sull'onda della logica, divenuta ineluttabile, del cambiamento pianificato ${ }^{7}$ : il mutamento non è più osservato, ma è (deve essere) guidato (Lepresle, 2003). Lo strumento principe di queste tendenze è costituito dal progetto che sembra pervadere ogni ambito del quotidiano: progetto professionale, di carriera, di formazione, di vita; ma questo essere proiettati in dinamiche di progettualità permanente presenta il rischio di vedere tramutare il progetto stesso in un escamotage che maschera fughe in avanti da un presente precario attraverso la costruzione utopica di un futuro certo e senza sbavature ma privo di legami con la storia intesa come biografia. Viene così a mancare il rapporto di continuità tra esperienza e progetto che consente una costruzione narrativa della propria identità, professionale nella fattispecie. Se pensiamo ai percorsi di formazione, una progettualità quale quella illustrata da Lepresle - che, sia detto per inciso, non vuole essere una critica generalizzata al progetto in quanto modalità di lavoro e didattica, quanto piuttosto un invito a farne un uso ponderato e critico - viene privata del suo contenuto pedagogico che dovrebbe essere proprio quello di legare, in un processo di continuità narrativa, esperienza vissuta e progetto integrando il secondo nella prima. Se per contro le esperienze passate, le conoscenze e le capacità accumulate perdono il loro significato e divengono inutili, cioè subito obsolete, in funzione di un progetto di professionalità spendibile in un mercato in continua trasformazione, se questo è vero, diviene difficile per l'uomo trovare una sua collocazione stabile nel presente che gli consenta di creare ponti di significato tra passato e futuro.

La portata di queste considerazioni non può lasciare indifferente il formatore e questo per almeno due aspetti: le persone in formazione - e in particolar modo gli adulti che si riqualificano - sono coinvolte in un momento di transizione (Perret-Clermont \& Zittoun, 2002) che comporta la ricostruzione della propria identità professionale; d'altro canto è la professione stessa di formatore a venire rimessa in discussione.

Per quanto riguarda il primo aspetto, è quasi inevitabile l'insorgere di momenti di smarrimento nei quali si rivela difficile mettere a fuoco il senso stesso del percorso intrapreso. La persona in formazione si trova nella situazione, per certi versi paradossale, di una maggior autonomia (grazie anche alla modularizzazione) ma con la necessità disporre di una guida. Al formatore, chiamato ad accompagnare percorsi di formazione il cui senso non risiede più solamente nell'acquisizione di conoscenze o competenze ma piuttosto nella ricostruzione di una propria realtà professionale e personale, il problema si pone a due livelli: l'uno attinente all'assistenza della persona in formazione, l'altro al processo formativo. 
Lui stesso, e siamo al secondo aspetto, è chiamato ad attribuire significati alla sua professionalità che cambia: le domande, i dubbi, lo smarrimento delle persone in formazione che accompagna, sono anche suoi, gli appartengono.

\section{Modularizzazione dei percorsi di formazione: un'evolu- zione non solo strutturale}

Entrando nel merito della problematica che ci interessa più da vicino, notiamo come la modularizzazione dei percorsi di formazione riproduca ed in parte amplifichi quei fenomeni di rottura della linearità che caratterizzano il mercato del lavoro. Un conto è infatti trovarsi all'interno di un percorso che contempla un inizio e una fine, in qualche modo ritualizzati o comunque riconoscibili al senso comune (ritrovo il tal giorno, alla tal ora, in un contesto che rinvia all'apprendere delle esperienze scolastiche, con un gruppo che rimarrà più o meno stabile; esami o comunque riti di certificazione che ne sanciscono la fine), in cui l'articolazione dei contenuti segue, di regola, una logica lineare per cui i legami tra quanto avviene prima e quanto poi contribuiscono in maniera quasi naturale alla costruzione progressiva di significato. Altro è orientarsi in percorsi in cui i nessi tra $\mathrm{i}$ vari contenuti non sono più dati ma vanno continuamente costruiti mettendo in relazione tra loro sequenze discrete, i moduli, (Lipari, 1995) più o meno indipendenti. Ognuna di queste sequenze costituisce un contesto nel contesto, con sue regole, logiche, tempi, specifici. Questo ci porta a porre il problema dell'articolazione cronologica dei percorsi modulari e del come i contenuti trattati nei vari moduli si integrino tra loro e trovino una loro coerenza rispetto agli obiettivi di un percorso. Le esperienze prese in esame mostrano come malgrado la modularità dei percorsi di formazione si voglia svincolata da logiche lineari, la realtà si discosti da questo quadro per certi versi ideale ${ }^{8}$; gli utenti necessitano di una logica che attribuisca significato ai singoli moduli rispetto al contesto. Appare insomma difficile affrancarsi da modalità di pensiero improntate ad un'esperienza lineare del tempo, perlomeno senza intervenire in maniera incisiva sulla struttura dei moduli e dei contenuti e quindi sugli aspetti pedagogici e didattici. L'articolazione dei percorsi non si risolve certamente nei suoi aspetti organizzativi.

La potenziale eterogeneità della strutturazione dei contenuti all'interno dei vari moduli e della maniera in cui questi vengono proposti quali oggetti di apprendimento pone due ordini di problemi: un primo dai risvolti epistemologici che concerne la frantumazione della conoscenza e un secondo attinente al rapporto tra contenuti e modalità didattiche.

Un approccio alla conoscenza articolato attorno a discipline ancorate a tradizioni accademiche più o meno stabili assicura di per sé una base di organicità, di strutturazione e di ordine del sapere. D'altro canto difficilmente questa opzione potrà rivelarsi funzionale alle esigenze specifiche di percorsi di formazione professionale, a maggior ragione se modulari, che mirano più alla costruzione di competenze, di capacità di agire in situazione, che alla semplice acquisizione di 
conoscenze. Il sapere si costituisce piuttosto attorno a tematiche specifiche o situazioni problema rendendo necessaria una sorta di estrapolazione di segmenti di sapere dalla disciplina di riferimento, estrapolazione che richiede la ricostruzione di una logica in grado di conferire loro uno spazio e un senso all'interno di un nuovo quadro di riferimento (il tema da affrontare e/o il profilo di competenza). L'assenza di un siffatto criterio di ordine conduce immancabilmente alla frantumazione della conoscenza e agli effetti che ne conseguono. Si pone quindi l'esigenza di trasporre contenuti tradizionalmente improntati ad una logica disciplinare finalizzandoli all'acquisizione di competenze, e questo senza incorrere nel rischio di un adeguamento acritico ai bisogni del mercato sovente foriero di processi di banalizzazione dei contenuti stessi.

Il rapporto tra contenuti e modalità didattiche, e siamo al secondo ordine di problemi, non è mai neutro. Determinati contenuti possono apparire in una connotazione peculiare e in una luce diversa a seconda del contesto didattico entro cui vengono presentati ed elaborati. Operare una scelta in rapporto ai contenuti significa anche stabilire le modalità attraverso le quali questi divengono oggetto di insegnamento (savoir à enseigner). Se, come sembra emergere dalle esperienze prese in esame e dalla vasta letteratura in materia, la formazione professionale mira all'acquisizione di competenze, la didattica deve essere necessariamente tesa al loro sviluppo.

Direttamente legate alla didattica, alcune problematiche legate alla valutazione meritano alcune considerazioni a parte:

a) Valutazione delle risorse elo competenze acquisite: il problema si pone in termini di adeguatezza delle modalità di valutazione in rapporto alle risorse rispettivamente competenze a cui si mira e alle modalità didattiche applicate.

b) Certificazione e riconoscimento: si tratta qui di rispondere ad un'esigenza di trasparenza resa ancor più impellente dalla liberalizzazione del mercato della formazione. Sia il mercato del lavoro che le persone che intraprendono un percorso di formazione necessitano di garanzie in ordine alla qualità e alla consistenza di certificati e diplomi. Si pensi in quest'ottica al sistema ECTS 9 o, nell'ambito della formazione professionale, al tentativo rappresentato dalla Centrale Svizzera dei moduli e al più recente dispositivo messo in atto dall'Unione europea con gli ECVET ${ }^{10}$.

Per entrambi gli aspetti si pone il problema, tuttora in larga misura irrisolto, di dar conto delle competenze acquisite in maniera informale, ovvero in luoghi non istituzionalmente deputati alla formazione.

La discontinuità dei percorsi modulari ha effetti anche sugli aspetti relazionali in quanto, a dipendenza della tipologia di percorso, la composizione del gruppo di persone in formazione può variare in misura più o meno marcata mentre $\mathrm{i}$ formatori di riferimento cambiano di regola da un modulo all'altro. Ne consegue che le relazioni tra pari e tra studenti e formatori assumono un carattere in qualche modo precario, penalizzante a vari livelli, non da ultimo quello della motivazione. 
Il contesto che abbiamo cercato, senza pretesa di esaustività, di tratteggiare costituisce il terreno nel quale gli attori dei percorsi di formazione modulare si muovono, negoziano e accompagnano percorsi i cui elementi non sono dati una volta per tutte ma, come in un puzzle in cui i pezzi possono essere incastrati in più modi che originano forme simili, ma mai uguali. Qui il formatore è chiamato a sostenere le persone in formazione a costruire senso e ad attribuirne egli stesso al proprio agire.

\section{I ruoli del formatore}

Il mutamento in atto nelle strutture e nei contesti della formazione nulla toglie alla centralità del ruolo del formatore, ma lo chiama a rivedere i suoi compiti e le sue competenze in relazione tanto ad ambiti direttamente attinenti alle istituzioni formative quanto all'azienda e agli spazi di transizione tra questa e la scuola. Soprattutto nel settore della formazione professionale, e più nello specifico della formazione continua di adulti, i punti di intersezione tra apprendimento e lavoro assumono una rilevanza tale per cui la riflessione sul ruolo del formatore non può esimersi dal considerarli quali luoghi del suo agire professionale a tutti gli effetti. Qui si situano le aree del transfert dalle risorse acquisite alle competenze agite e dell'apprendimento in contesti informali.

Ci sembra interessante, prima di entrare nello specifico della formazione modulare, operare un'incursione sul terreno della formazione in azienda del quale, perlomeno a livello di terminologia, i sistemi formativi sono più o meno debitori. Malgrado le differenze anche sensibili che si riscontrano nella letteratura, due figure professionali emergono più di altre: il coach e il tutor.

\section{Il coach}

Di più recente applicazione in ambito sportivo, il termine stava in origine a designare il Coachman, ossia il cocchiere, colui che ha il compito di condurre i cavalli in maniera sicura e veloce a destinazione. Per taluni (Reggiani, 2000) il coach è un superiore gerarchico che si occupa direttamente dell'apprendimento delle persone che gli sono sottoposte sia in rapporto ai contenuti che ai processi di apprendimento. In area tedesca una vasta letteratura - sovente assimilabile alla manualistica - mette l'accento ora sulle capacità di conduzione da un punto di vista psicologico, ora sulle attività di consulenza (Hilfe zur Selbsthilfe; König \& Volmer, 2003, p. 11). Altrove il coach designa la persona di fiducia con funzioni di collega esperto, o ancora un professionista esterno, anche esterno all'azienda, nel sostegno a personale dirigente (Jüster, Hildebrand \& al., 2002).

\section{Il tutor}

La figura del tutor ha le sue origini in ambito giuridico e designa colui al quale è 
affidata la cura degli interessi di una persona - giovane o non in grado di badare a sé stessa - sovente includendo la sua educazione. Dal contesto aziendale dove, secondo Reggiani, coordina i percorsi di formazione e di carriera delle persone con potenziali di crescita, il tutor entra nel contesto dei sistemi formativi designando sovente l'accompagnatore tout court, come ad esempio nella ricerca ATLASS $^{11}$ (Glikman, 2002).

Altre figure professionali ricorrono anche se con minor frequenza: in Reggiani troviamo il mentor ${ }^{12}$, che richiama il medioevale maestro di bottega, mentre nella letteratura tedesca si incontra il Lernbegleiter (Ghisla \& Zgraggen, 2004), dove l'accento è esplicitamente posto su un accompagnamento personale dei processi di apprendimento.

Da questo breve excursus nella realtà aziendale ci sembra di poter estrapolare due considerazioni significative nell'ottica della nostra analisi: l'una attiene ai luoghi della formazione ${ }^{13}$ costituiti perlopiù da contesti informali, mentre la seconda riguarda il focus dell'attività dei formatori, posto non tanto sull'insegnare (nel senso di trasmettere conoscenza) quanto piuttosto sull'accompagnare le persone in formazione lungo percorsi di acquisizione di competenze.

Si tratta di caratteristiche che ritroviamo entrambe nel contesto del Progetto Poschiavo dove emerge il ruolo chiave del formatore quale cerniera tra momenti formativi e attività pratica.

Entrando nello specifico dei percorsi modulari, abbiamo avuto modo di mettere in evidenza l'ambiguità che li caratterizza (potenziale di flessibilità e versatilità versus rischio di smarrimento e perdita di senso) evidenziando i problemi che $\mathrm{i}$ formatori sono chiamati ad affrontare:

- frantumazione dei percorsi di formazione;

- questione epistemologica dell'approccio alla conoscenza e impostazione didattica;

- modalità di certificazione e di valutazione;

- esigenza di ricostruire un senso in rapporto a un percorso evolutivo;

- problematiche legate alla dimensione relazionale.

Questi aspetti si declinano in maniera anche molto diversa a dipendenza della tipologia di percorso modulare. Le esperienze condotte presso l'ISPFP (Bausch \& Ghisla, 2005) ${ }^{14}$ e il confronto con la letteratura ci portano a collocare i vari tipi di percorso su di un continuum che vede agli estremi:

a) Percorsi predefiniti; strutture con una logica interna al sistema, composte da moduli consecutivi e funzionali ad un profilo in uscita predeterminato.

b) Percorsi ad hoc, costituiti da moduli sparsi, costruiti in funzione delle esigenze delle singole persone in formazione e che presentano quindi logiche esterne al sistema.

Il ruolo del formatore muta di conseguenza anche sensibilmente a dipendenza del contesto nel quale si trova ad operare; alcune tendenze sono nondimeno 
emerse dalle nostre analisi e in particolare il fatto che la professione di formatore non possa più limitarsi al ruolo classico del docente (inteso come l'esperto che insegna una disciplina o un argomento specifico) per abbracciare attività attinenti alla gestione della struttura e delle persone coinvolte nonché all'accompagnamento con tutte le sfaccettature che lo caratterizzano. Abbiamo identificato tre gruppi di funzioni ognuna delle quali può essere messa in relazione con un ruolo più o meno definito: gestione, docenza e accompagnamento.

\section{Gestione (management) 15}

In senso lato la gestione (management) concerne l'organizzazione dei percorsi di formazione modulari. Il ruolo di manager appare tendenzialmente più incisivo quando i percorsi presentano una struttura predefinita mentre avvicinandosi alle tipologie che abbiamo definite ad hoc, molte delle sue funzioni tendono a ricadere sulle spalle delle singole persone in formazione o a venir assunte da consulenti (in percorsi di carriera, ecc.) esterni al sistema.

Vediamo di seguito queste funzioni in maniera più differenziata.

\section{Articolazione dei percorsi}

A chi è chiamato a gestire un percorso di formazione spettano compiti che vanno dalla scelta dei contenuti, alla loro traduzione in attività di formazione, all'articolazione coerente dei vari moduli. Il presupposto imprescindibile all'espletamento di queste funzioni è costituito dalla definizione chiara di un profilo di competenza in uscita e questo implica che il gestore della formazione partecipi direttamente a questa definizione - nel caso di professioni innovative come ad esempio il WPM o l'RPO per i quali è stato necessario un lavoro di ricerca e di analisi delle competenze richieste nei diversi contesti professionali - oppure, nel caso di profili consolidati, che proceda a una verifica periodica dell'adeguatezza del profilo al contesto economico e sociale di riferimento.

\section{Gestione della rete di relazioni}

I percorsi modulari realizzati presso l'ISPFP mostrano l'essenzialità della funzione di trait d'union, di mediatore tra $\mathrm{i}$ vari attori. In particolare emerge l'importanza di creare occasioni di incontro tra docenti e accompagnatori in modo da favorire la coerenza e l'integrazione dei contenuti dell'azione formativa. Più in generale, si tratta di promuovere l'evoluzione di un percorso fisiologicamente suddiviso in sequenze più o meno indipendenti in una struttura reticolare in cui i vari attori sappiano muoversi di concerto.

Uscendo dai confini di un singolo percorso di formazione, una rete di relazioni efficace consente al gestore di scegliere in maniera ottimale le risorse - docenti ad esempio - in funzione del contesto di riferimento e di porsi quale intermediario privilegiato tra i diversi luoghi dell'apprendimento, istituzioni formative e azienda in primo luogo. In corsi come RPO, AP-CmC o WPM dove l'integrazione tra apprendimento e attività pratica è parte integrante del per- 
corso, queste reti si sono dimostrate essenziali. Le stesse considerazioni erano già scaturite nell'ambito del Progetto Poschiavo dove la rete di relazioni con diversi centri di competenza ha consentito di rispondere alle domande di consulenza specialistica provenienti dai progetti di sviluppo. La ricerca ha inoltre messo in evidenza la necessità di aggiornare continuamente questa rete di collaborazioni in funzione non solamente dei bisogni contingenti ma anche di quelli potenziali (non ancora esplicitati) che emergono dalle analisi dei terreni di applicazione dei progetti di formazione.

\section{Supervisione}

In quanto beneficiario di una visione globale del sistema, il gestore è chiamato a garantire la coerenza tra modalità dell'azione formativa e obbiettivi del percorso. In questo senso va inteso il lavoro di supervisione del lavoro di docenti e accompagnatori che può esplicarsi secondo modalità diverse: il gestore può partecipare in prima persona a tutte le attività di formazione oppure limitarsi ad interventi puntuali. In questo secondo caso si rivela indispensabile la messa a punto di documenti di riferimento comuni a tutti i docenti - quali, ad esempio, un protocollo didattico che contribuisca ad assicurare uno standard didattico minimo. Importante è pure il coordinamento dell'azione degli accompagnatori alfine di garantire un'integrazione e un interscambio delle competenze specifiche di cui ognuno di questi è portatore. Nell'esperienza WPM ad es. si è creato un team gestito dal management che ha favorito uno scambio di esperienze del quale, in ultima analisi, le persone in formazione hanno potuto beneficiare. La ricerca sul Progetto Poschiavo o il già citato rapporto sulla ricerca europea ATLASS confermano l'importanza che competenze complementari possano essere messe tra loro in relazione attraverso un lavoro di équipe.

\section{Valutazione di profili di competenza}

Senza voler entrare nel merito della problematica relativa alla costruzione di bilanci delle competenze (Selvatici \& Grazia, 2004 e Camus, 2003), ci preme sottolineare come il manager debba essere in grado di analizzare domande di ammissione composte da dossier personali anche molto eterogenei tra loro. Questo implica che conosca il mercato della formazione e sia quindi in grado di valutare in merito alla concessione di equivalenze e, più in generale, alla costruzione di percorsi individuali. Si pensi ad esempio alla già accennata problematica della valutazione di competenze apprese in contesti informali.

Muovendo dalle funzioni appena discusse, le competenze di cui un manager di corsi di formazione modulare deve disporre si possono articolare attorno a due punti focali:

a) La comunicazione e la negoziazione.

Essendo di regola i moduli proposti da istituzioni diverse (aziende, università, altre istituzioni di formazione) o provenienti da diverse aree culturali (lingua, 
tradizioni pedagogiche, ecc.), al manager sono richieste doti di mediazione che, fatti i dovuti distinguo, possono essere paragonate a quelle proprie al testimone culturale di estrazione antropologica. Diverso è infatti negoziare una collaborazione con uno specialista attivo in un'azienda piuttosto che con un professore universitario: diversi i valori culturali di cui sono portatori, diversi $\mathrm{i}$ linguaggi, diverse le attese e le rappresentazioni in rapporto alla formazione. Questo aspetto emerge ad es. con chiarezza dalla ricerca sul Progetto Poschiavo in relazione sia alla gestione che all'accompagnamento.

b) L'approccio alla ricerca applicata.

Chi gestisce percorsi di formazione deve essere in grado di agire all'interno di una dimensione di ricerca permanente che gli consenta di analizzare le esperienze in modo da poterle adattare a contesti diversi. Questo richiede perlomeno tre competenze essenziali: operare secondo un approccio di pratica riflessiva, saper operare un transfer continuo dal terreno dell'azione formativa a quello della concettualizzazione e disporre di strumenti di lettura del contesto che sappiano andare oltre la semplice raccolta dei bisogni per entrare nel merito dell'analisi delle dinamiche delle organizzazioni (Lipari, 1995).

I momenti salienti del lavoro del gestore si possono riassumere nella definizione dei percorsi (lettura e interpretazione dei contesti, costruzione di percorsi funzionali alle esigenze del mercato del lavoro ma anche dei potenziali partecipanti alla formazione, ecc.) e nella gestione vera e propria dove è chiamato ad amalgamare i vari pezzi di un percorso alfine di tenerlo unito e dotato di una certa coerenza: agire in qualche misura da forza centripeta per controbilanciare una struttura che di per sé presenta spiccate tendenze centrifughe ${ }^{16}$.

\section{Docenza}

Si tratta delle funzioni attribuite di regola ad un docente ovvero al ruolo che più si avvicina all'insegnante in senso classico e per questo, dei tre che abbiamo preso in considerazione, quello più facilmente identificabile con una figura professionale riconosciuta.

Come per gli altri profili, le funzioni che un docente assume dipendono in larga misura dal contesto e in particolare dalla tipologia del percorso modulare. Oltre che alla padronanza del suo sapere specifico (disciplina o contenuti tematici: cfr. cap. 1.1), gli viene richiesto uno sforzo di strutturazione di questo sapere tale da attribuirgli un significato adatto alle peculiarità dei diversi contesti. Le condizioni necessarie a soddisfare quest'esigenza sono perlomeno due: beneficiare di una visione d'insieme del percorso formativo e del profilo di competenza in uscita e disporre di strumenti didattici adeguati e coerenti con la logica del sistema. L'assunzione di responsabilità in questo senso può esplicarsi in diversi modi:

a) Il docente può assumerle in prima persona partecipando, ad esempio, alla definizione e alla negoziazione del profilo professionale e/o all'impostazione del 
percorso. Gli sarà in questo caso richiesto un impegno che travalica i confini del suo modulo. Nel corso WPM, ad esempio, un docente ha gestito, accanto alla sua attività di esperto, incontri regolari durante tutto l'arco del percorso di formazione volti a favorire l'integrazione dei contenuti dei vari moduli nel profilo professionale.

L'impegno supplementare richiesto e i relativi costi, nonché, talvolta, la scarsa motivazione dei docenti ad investire risorse in un processo le cui ricadute non sono evidenti, rende questa opzione difficilmente praticabile.

b) Egli può adeguarsi alla logica del sistema; in questo caso non gli viene richiesto di partecipare alla costruzione di questa logica ma di prenderne coscienza e impostare la sua azione formativa di conseguenza.

c) La terza opzione porta il docente a concentrarsi esclusivamente sulla trasmissione del suo sapere specifico demandando ad altri (accompagnatori, persone in formazione) qualsiasi sforzo di integrazione. Malgrado le controindicazioni in termini di attribuzione di significato, ci sembra questa la tendenza destinata a prevalere, in quanto indotta dalla struttura modulare stessa.

Le tre opzioni si pongono idealmente in relazione direttamente proporzionale con il continuum sul quale abbiamo situato le tipologie di percorsi modulari: più la logica è interna al sistema maggior dovrebbe essere lo sforzo dei docenti nel contestualizzare il loro apporto, mentre quando i moduli sono scelti in funzione degli interessi delle singole persone in formazione, tale lavoro si rivela difficile a causa dell'eterogeneità delle singole esigenze.

Più ci si avvicina alla terza delle opzioni prese in considerazione meno il docente avrà un rapporto diretto e continuato con le persone in formazione, accrescendo la distanza tra studenti, contenuti e percorso di formazione che la struttura modulare stessa tende a generare. In riferimento al triangolo pedagogico proposto da Houssaye e ripreso da Aumont e Mesnier (1992), ci troviamo di fronte ad un caso emblematico di terzo escluso ${ }^{17}$ : nell'intreccio di relazioni tra sapere, insegnate ed allievo è la relazione tra gli ultimi due - insegnante ed allievo - a venire in qualche modo sacrificata. Ci si può porre la domanda se la figura del docente si vada sempre più identificando con il sapere di cui è portatrice oppure se la tendenza sia piuttosto quella di un recupero degli aspetti relazionali e dei processi di apprendimento.

Indipendentemente dal contesto in cui opera, il docente è comunque chiamato a rivedere il proprio ruolo. Accanto alle competenze più evidenti e facilmente riconoscibili e riconosciute - ovvero di depositario di un capitale di conoscenze - se ne vanno ad affiancare altre che Meyrowitz (1993) chiama di retroscena. Pensiamo qui a due possibili declinazioni di questo retroscena: da un lato l'affermarsi di forme di apprendimento che esulano dal palcoscenico per eccellenza di chi insegna - l'aula -, quali l'apprendimento autonomo o la progettualità, dall'altro la dimensione soggettiva del formatore in quanto egli stesso soggetto che apprende, che cioè trasforma e integra continuamente il suo sapere. 
Crediamo siano, questi, ambiti degni di essere indagati in quanto sovente poco accessibili ai docenti stessi. Huber e Chautard (2001) parlano di «savoir cachés des enseignants» individuando nelle metodologie di analisi del lavoro (approcci biografici ma anche metodologie mutuate dalla psicologia del lavoro) una possibile via che consente di farle emergere. Di fronte a queste metodologie - e sono gli stessi autori ad indicarlo - i docenti si trovano sovente a disagio: "Pour eux [gli insegnanti] les seuls savoirs professionnels dont ils sont conscients résident dans la maîtrise de leur(s) discipline(s). L'existence de savoirs professionnels transversaux [...] est faiblement perçue. Il y a là une prise de conscience à susciter» (ibid., pp. 144-145). Hüberman (1992) parla dal canto suo di una dimensione soggettiva del formatore da riconoscere e valorizzare attraverso un processo dialettico che coinvolge il formatore stesso e un ricercatore (o formatore di formatori ${ }^{18}$. Entrambi gli approcci attribuiscono alla dimensione soggettiva del professionista un valore scientifico utile alla comprensione del mestiere di formatore $^{19}$; la ricerca sul Progetto Poschiavo costituisce un esempio di applicazione di tale assunto

E ipotizzabile che la valorizzazione di questi aspetti possa contribuire alla ricomposizione dello strappo relazionale tra docenti e persone in formazione recuperando la dimensione esclusa del triangolo pedagogico: si tratta infatti di competenze necessarie ad entrambi gli attori e i docenti possono, esplicitandoli, fare dei loro savoirs cachés una risorsa accessibile a chi apprende.

\section{Accompagnamento}

Quanto finora osservato, in relazione anche alla formazione in azienda, attribuisce a al ruolo di accompagnamento un'importanza crescente. Esso si contraddistingue per una marcata attenzione alle risorse metodologiche, in particolare in rapporto a tre aspetti: a) le modalità di apprendimento e l'utilizzo di strumenti innovativi, b) l'integrazione di competenze e c) gli aspetti relazionali.

a) Le esperienze hanno dimostrato come l'impiego di strumenti di apprendimento quali le piattaforme o, più semplicemente, l'e-mail, i forum o simili non possa essere considerato acquisito a priori: occorre al contrario che si generi un'abitudine al loro impiego quando non una vera e propria formazione specifica di cui la componente tecnica è uno - e nemmeno il più importante - dei fattori che entrano in linea di conto. L'accompagnamento deve, in questo caso, sostenere la persona in formazione nell'integrazione di queste tecnologie nei processi di apprendimento portandola a riflettere sui limiti e sui vantaggi così come sugli aspetti cognitivi e socio-cognitivi soggiacenti.

La capacità autoreferenziale di pensare ai propri processi di apprendimento costituisce quella che potremmo definire l'altra faccia della medaglia della formazione: apprendere ad apprendere (e quindi acquisire coscienza dei propri processi, strategie, ecc.), saper operare coscientemente transfert di competenze, sapersi muovere all'interno delle dinamiche dell'apprendimento auto- 
nomo, sono altrettanti ambiti, attinenti ai processi metacognitivi, di cui l'accompagnamento dovrebbe farsi carico.

b) Sebbene in maniera non esclusiva, spetta in buona misura all'accompagnatore sostenere gli studenti nei processi di attribuzione di significato ai saperi e alle capacità trattate nei vari moduli e questo integrandoli nei profili di competenza mirati, siano essi predefiniti o selezionati ad hoc dalle persone in formazione. Tale integrazione necessita di una riflessione critica costante e di momenti di negoziazione imperniati attorno ai profili di competenza oltre che ai processi di attribuzione di senso. Una sorta di percorso formativo all'interno del corso che si è rivelato tanto più efficace - nelle esperienze prese in esame - quanto più legato alla pratica intesa come luogo in cui agire le competenze (stage, progetti, ecc.).

c) Percorsi personalizzati, apprendimento autonomo, continui mutamenti dei setting formativi costituiscono altrettanti fattori che ostacolano la creazione di un gruppo di studenti coeso con il conseguente pregiudizio al potenziale di costruzione sociale della conoscenza. Curando le relazioni tra studenti e tra questi e gli altri attori (gestione, docenti, altre risorse), le funzioni di accompagnamento possono favorire l'implementazione di dinamiche di collaborazione sia mettendo in contatto studenti che hanno interessi convergenti o complementari, sia, nell'ambito delle tecnologie informatiche, favorendo la creazione di gruppi virtuali. A questo proposito ci sembra interessante notare come le tecnologie della comunicazione presentino aspetti ambigui: potenzialmente sono in grado di sopperire alla mancanza di continuità relazionale tipica di molti percorsi modulari (cfr. articolo di Botturi \& Cantoni in questo numero), d'altro canto, se non adeguatamente sostenute, possono aumentare la distanza sociale o comunque rivelarsi inefficaci in termini di apprendimento (Calvani \& Rotta, 2000).

Il terreno d'azione per eccellenza dell'accompagnatore è quindi costituito dai processi di apprendimento di persone con le quali intreccia relazioni perlopiù impostate su di un rapporto di fiducia. Egli è la figura di riferimento chiamata ad aiutare gli studenti ad orientarsi nei loro percorsi. Inevitabilmente le competenze che emergono come indispensabili riguardano le dimensioni relazionale e metodologica, dalla conoscenza degli strumenti didattici - tecnologici in particolare - alla gestione degli aspetti amministrativi, dalla creazione di occasioni di incontro alla gestione di colloqui fino alla cura dei processi di apprendimento. Il quadro che scaturisce dall'analisi delle esperienze dei corsi modulari presi in esame è sostanzialmente confermato dalla ricerca sul Progetto Poschiavo e da ATLASS che mette in evidenza, tra le altre, la dimensione affettiva della relazione di accompagnamento.

Glikman (2002) segnala come non esista un buon modello di accompagnatore ${ }^{20}$ ponendo tra le competenze indispensabile alla sua azione formativa la flessibilità di pratiche e la capacità di adeguarsi - e quindi di saperli interpretare - a 
contesti diversi. Nella ricerca sul Progetto Poschiavo si parla d'altro canto della "capacità di reinventarsi ogni volta una professione» (Bausch, Cattaneo \& Schürch, 2000).

Le competenze da retroscena accomunano, sebbene in ambiti e con modalità di applicazione diverse, gestori e accompagnatori e quindi, le competenze emerse per i primi riguardano anche i secondi ad un livello che potremmo definire più micro in quanto quest'ultimi beneficiano di regola di rapporti più diretti con le persone in formazione. Anche loro sono chiamati a mediare e negoziare e, dovendosi «reinventare ogni volta la professione» ad adottare strategie e metodi di pratica riflessiva, quando non di attitudine alla ricerca applicata.

Per entrambi si pone un problema di identità: se la loro azione formativa prescinde da un rapporto diretto con dei contenuti da trasmettere, su quali basi possono diventare degli interlocutori autorevoli? Come possono ricollocarsi dentro una dimensione di memoria storica garante di un legame con la tradizione e quindi con rappresentazioni che vincolano l'insegnante a una categoria ben precisa di sapere, solitamente disciplinare? Come far fronte dunque a rappresentazioni del formatore (ancora) molto ancorate all'immagine del docente?

Gli accompagnatori in particolare si trovano a dover costantemente negoziare un ruolo che sovente esula dalle rappresentazioni, e quindi dalle attese, delle persone in formazione. È quanto emerge in particolare dalla ricerca sul Progetto Poschiavo dove gli accompagnatori hanno implicitamente negoziato con le persone in formazione un ruolo di esperti in tecnologie della comunicazione previsto in origini quale supporto metodologico; si sono cioè attribuiti un sapere specifico che ha permesso loro di essere riconosciuti in quanto formatori. Nel corso WPM - l'unico tra quelli presi in esame a contemplare un accompagnatore che non assumesse contemporaneamente altre funzioni - il tentativo di attribuire ad un docente il compito di coordinare le attività di integrazione dei contribuiti dei diversi moduli ha tendenzialmente messo in ombra la figura dell'accompagnatore forse perché - e l'ipotesi tenderebbe a confermare la fragilità del profilo nelle rappresentazioni correnti - meno autorevole del docente.

\section{Conclusion $i$}

La modularizzazione dei percorsi di formazione comporta ripercussioni che vanno oltre gli aspetti strutturali e organizzativi interessando direttamente sia l'approccio alla conoscenza e le modalità didattiche che il ruolo del formatore. L'ambiguità insita nelle strutture modulari che affiancano flessibilità (quindi maggiore libertà nella gestione dei percorsi formativi) e caratteristiche di discontinuità e frammentazione, mette lo studente in difficoltà e rende pertanto auspicabile, se non necessario, un supporto che gli permetta una reale attribuzione di senso. Ed è proprio in quest'ottica che le diverse figure di formatore sono chia- 
mate ad espletare la loro azione formativa che per altro si concentra sulle dimensioni metodologica e relazionale. Le funzioni che caratterizzano i tre profili emersi - gestore, docente e accompagnatore - possono combinarsi in maniera diversa in funzione delle situazioni contingenti e in particolare della tipologia di percorso modulare. Questo ci pone di fronte ad una serie di interrogativi relativi ai processi di costruzione dell'identità professionale: se la tendenza emergente è quella di una suddivisione del lavoro che vede il docente sempre più esperto in un determinato ambito di conoscenza e sempre meno direttamente implicato nei processi di apprendimento, il gestore sempre meno formatore e più manager e un accompagnatore professionista dedito ad un supporto che - oltre ad aspetti strettamente metodologici - coinvolge la sfera personale, quali sono le rappresentazioni e attese indotte nelle persone in formazione? Quale la percezione, in termini di identità, che il formatore può avere di se stesso e della propria professionalità?

Le esperienze e le ricerche prese in considerazione sembrano far emergere la necessità di ridurre anche in quest'ambito la frammentazione integrando i vari ruoli attraverso la collaborazione tra le varie figure e valorizzando la dimensione che abbiamo definita soggettiva del formatore. Forse solamente in questo modo è possibile dar seguito alla legittima esigenza dei formatori - qualsiasi sia il loro ruolo - di essere riconosciuti come professionisti e come soggetti con una loro identità.

\section{Note}

1 Al tema dell'apprendimento in situazione è stato dedicato il Nr. 3 del 2001 «Eclairages sur la «cognition située» et modélisation des contextes d'apprentissage» di questa rivista.

2 Tra le molte definizioni possibili e applicate ci sembra che alcuni elementi comuni possano essere identificati. A partire da Ryle che, già nel 1949, vedeva nel concetto di competenza una sorta di ponte tra sapere teorico e sapere pratico (cfr. Frega, 2001), tutti gli autori sembrano concordi nel mettere in relazione la competenza con l'azione sul terreno. Questa si connoterebbe per la capacità di integrare «saperi» in funzione di un'attività. Tra le varie correnti alcune mettono l'accento piuttosto sulla disposizione ad agire, altre invece sul fatto che la competenza si da solo in azione, altri ancora integrano i due aspetti (azione e disposizione) vincolando l'agire competente a una situazione intesa come contesto nel quale mobilitare le risorse di cui un attore dispone. Il problema si pone, da un punto di vista pedagogico e didattico, sul come la competenza diventi oggetto dei processi di apprendimento. Molto utile in questo senso la definizione di LeBoterf (1994) che la scompone in tre fattori (sapere, saper fare e saper essere), fattori che, seppur con declinazioni e accenti diversi costituiscono una piattaforma di riferimento riconosciuta. Confronta anche Ghisla (2002).

3 L'ISPFP è un istituto dell'Ufficio Federale per la Formazione professionale e la Tecnologia (UFFT).

4 Si veda per un descrittivo dei percorsi il sito www.ispfp.ch e per un'analisi differenziata Bausch \& Ghisla, 2005.

5 Il Progetto Poschiavo (PP), avviato nel 1996 su iniziativa dell'ISPFP, e sostenuto dall'UFFT, da Swisscom e dalla Jacobs Foundation nonché dai cantoni Grigioni e Ticino, si prefiggeva di coniugare formazione, comunicazione mediata dal computer e sviluppo re- 
gionale sostenibile attraverso l'elaborazione e la realizzazione di progetti di sviluppo. I promotori dei progetti beneficiavano dell'accompagnamento di un Assistente di Pratica in Formazione a Distanza (APFD), figura di formatore che può essere considerata il capostipite di profili professionali innovativi più o meno direttamente legati alla formazione abbinata all'impiego delle tecnologie della comunicazione di cui l'ISPFP è stato promotore.

6 Richieste FNS N. 5004-51723 (Prof. A.-N. Perret-Clermont, Institut de psychologie, Università de Neuchâtel); N. 5004-47955 (Prof. D. Peraya, Tecfa, Université de Genève) e N. 5004-4796 (D. Schürch, ISPFP, Lugano). In particolare prenderemo in esame la parte della ricerca condotta dall'ISPFP sugli APFD incentrata su una serie di interviste condotte durante l'arco della formazione (Bausch, Cattaneo \& Schürch, 2000).

7 Ma quale cambiamento? Ci troviamo di fronte a un paradosso: panificazione e progetto (e quindi orientamento al futuro) che però tendono a proiettare - e quindi a perpetuare schemi del presente all'interno di dinamiche che non sono evolutive ma piuttosto di frammentazione della linearità narrativa. Sul tema del rapporto tra passato, presente e futuro nella società della comunicazione e sui suoi effetti in rapporto ai processi di costruzione dell'identità contemporanea, rinviamo al testo di Merlini (2004) in cui l'autore sviluppa, in modo particolare nel primo capitolo, il tema dell'esperienza del tempo nella società della comunicazione mettendo in evidenza come questo sia divenuto un tempo discontinuo che mette in crisi la linearità cumulativa propria della temporalità storicista.

8 Ideale in quanto risponde, in linea teorica, in maniera ottimale sia alle esigenze di flessibilità, sia alla prospettiva epistemologica di un sapere disposto in rete. I percorsi dovrebbero poter essere costruiti ad hoc dalle persone in formazione scegliendo i moduli, in un mercato sul quale operano diversi offerenti legittimati, in funzione di criteri individuali (luogo, tempi, affidabilità dell'offerente, ....). La disposizione reticolare del sapere dovrebbe, d'altro canto, permettere di acquisire porzioni di conoscenze e/o competenze tra loro integrabili in maniera da soddisfare le esigenze più diverse.

9 ECTS: European Credit Transfer System. Per una descrizione del sistema ECTS rimandiamo a: http://europa.eu.int/comm/education/programmes/socrates/ects_it.html

10 ECVET: European Credits transfert system for Vocational Education and Training. Si veda il documento della Commissione Europea per l'Educazione e la Cultura all'URL: http://www.na-bibb.de/uploads/leo/ecvet_eu-kommission_principles.pdf

11 Supporting Adult Learners To Achive Success. Si tratta di una ricerca comparata sulle attività tutoriali che ha coinvolto il NFER (Fondazione nazionale inglese per la ricerca in educazione), l'INRP (Istituto nazionale di ricerca pedagogica) francese e, per la Germania, il LSW (Istituto della Wesfalia del nord per la scuola e la formazione degli adulti).

12 Le origini del termine - che ha subito un curioso processo di anglicizzazione - vanno ricercate nella mitologia greca: Mentore era il precettore incaricato da Ulisse, in partenza per la guerra di Troia, di vegliare sull'educazione del figlio Telemaco.

13 Sull'argomento si vedano i vari contributi nel testo curato da Amietta (2000) e, specificatamente sull'apprendimento in azienda, Dehnbostel (1998)

14 Cfr. il contributo di M. Pilz in questo numero e anche Kloas (1999):

15 In questa sede abbiamo voluto concentraci su tematiche più o meno direttamente connesse ad aspetti pedagogici e didattici. Ci sembra però doveroso evocare alcune delle funzioni amministrative e organizzative in quanto essenziali al funzionamento di un percorso modulare: a) la gestione finanziaria, complessa in ragione dell'articolata struttura amministrativa e dal numero generalmente elevato di attori coinvolti; b) la gestione amministrativa (contratti, accordi, organizzazione pratica dei momenti formativi, ecc.); c) la gestione della rete di collaborazioni.

$16 \mathrm{Ci}$ sembra interessante un confronto tra il profilo di gestore emerso dalle nostre indagini e quello di ingegnere della formazione. Si vedano a questo proposito, per la letteratura in lingua italiana, il già citato testo di Lipari (1995) o del gruppo di lavoro dell'ISPFP di Lu- 
gano (Cattaneo et al., 2000); per le opere in francese, LeBoterf (1999) o Pain (2003). La rivista Education Permanente ha dedicato il Nr. 157, Vol 4 «Où en est l'ingénierie de la formation?» del 2003 al tema..

17 Secondo l'autore, ognuna delle tre attività (insegnare, apprendere, formare) che legano i poli del triangolo pedagogico (sapere, insegnante, studente) tende ad escludere uno dei detti poli: nell'attività dell'insegnare si tende ad escludere lo studente, nell'apprendere il rapporto privilegiato di instaura tra studente e sapere e nel formare (accompagnare in percorsi di formazione) è il sapere a venir messo in disparte.

18 Huberman (1992) parla di modello dei due cerchi per spiegare come la scarsa propensione degli insegnanti al cambiamento generi una sorta di cortocircuito del sistema che induce il docente stesso a reiterare le proprie pratiche didattiche. Secondo l'autore il problema può essere risolto invitando l'insegnante a ricostruire i processi cognitivi che soggiacciono alla sua attività formativa. Questa ricostruzione dell'attività professionale - attraverso una dinamica dialettica con un ricercatore -, implica da un lato un processo di presa di coscienza e dall'altro gratifica l'insegnante non solamente riconoscendo quella parte della sua attività solitamente negletta ma elevandola a oggetto di ricerca. L'interazione di questi due cerchi (attività pratica e ricostruzione-simulazione) tenderebbe ad instaurare un doppio circolo virtuoso all'interno del quale il passaggio continuo dal terreno dell'azione a quello della metacognizione favorirebbe le dinamiche di cambiamento. L'insegnante si sentirebbe compreso dal ricercatore e accetterebbe da questi stimoli in termini di consulenza, documentazione, proposte.

19 In rapporto alle teorie soggettive rinviamo a Lauken (1974), che può esserne considerato il capostipite, o Dann (1994). Per gli approcci autobiografici citiamo tra gli altri Demetrio (2002).

20 Nella ricerca ATLASS si parla di Tutor confermando la confusione terminologica che accompagna la designazione delle varie figure di accompagnatore.

\section{Bibliografia}

Amietta, P. L. (2000). I luoghi dell'apprendimento. Milano: F. Angeli.

Aumont, B. \& Mesnier, P.-M. (1992). L'acte d'apprendre. Paris: Presses Universitaires de France.

Bausch, L. \& Ghisla, G. (2005). Le esperienze modulari presso l'ISPFP (Rapporto finale). Lugano: ISPFP.

Bausch, L., Cattaneo, A. \& Schürch, D. (2000). La formazione degli APFD (Rapporto di ricerca FNR n. 5004-51723). Lugano: ISPFP.

Calvani, A. \& Rotta, M. (2000). Fare formazione in Internet. Manuale di didattica online. Trento: Erickson.

Camus, O. (2003). Le bilan de compétences: appropriation d'informations sur soi ou mise en conformité de l'autoreprésentation? Education permanete, 2, (155), 119-137.

Cattaneo, A., Messi, G., Schürch, D. \& Bausch, L. (2000). L'ingegneria dell'innovazione pedagogica (Rapporto di ricerca FNR n. 5004-51723). Lugano: ISPFP.

Dann, H.-D. (1994). Pädagogisches Verstehen: Subjective Theorien und erfolgreiches Handeln von Lehrkräften. In K. Reusser \& M. Reusser-Weyeneth (Ed.), Verstehen (S. 163182) Bern: H. Huber Verlag.

Dehnbostel, P. (1998). Lernorte, Lernprozesse und Lernkonzepte im lernenden Unternehmen aus berufspädagogischer Sicht. In P. Dehnbostel, H.-H. Erbe \& H. Novak (Ed.), Berufliche Bildung im lernenden Unternehmen (S. 175-194) Berlin: Sigma.

Demetrio, D. \& Alberaci, A. (2002). Istituzioni di Educazione degli adulti. Il metodo autobiografico. Milano: Guerini scientifica.

Frega, R. (2001). Conoscenza e competenza: alcune suggestioni in una prospettiva filosofica. 
Professionalità, Rivista di cultura, esperienze e innovazione per la formazione al lavoro, 65, 918.

Ghisla, G. \& Zgraggen, B. (2004). Swisscom vot-Story. Ein Modell innovativer betrieblicher Ausbildung Wissenschaftliche Begleitung, Schlussbericht. Lugano: USI.

Ghisla, G. (2002). Competenze: aspetti della discussione a livello internazionale [Manoscritto]. Lugano: ISPFP.

Glikman, V. (2002). Apprenants et tuteurs: une approche européenne des mediations humaine. Education permanete, 3, (152), 55-69.

Huber, M. \& Chautard, P. (2001). Les savoirs cachés des enseignants. Paris: L'Harmattan.

Huberman, M. (1992). De la recherche à la pratique: comment atteindre des retombées "fortes»? Revue française de pédagogie, 98, 69-81.

Hutchins, E. (1994). Comment le "cockpit» se souvient de ses vitesses? Sociologie du travail 4, 451-473.

Jüster, M., Hildebrand, C.-D. \& Pezold Hilarion, G. (2002). Coaching in der Sicht von Führungskräften - eine empirische Untersuchung. In C. Rauen (Ed.), Handbuch Coaching (S. 45-66). Göttingen: Hogrefe.

Kloas, P. W. (1999). Modulare Ausbildung im Rahmen des Berufskonzeptes: Reformansätze in Deutschland. Education permanente, Panorama, (Dossier EP 99/2, Panorama 99/3), 56-59.

Lauken, U. (1974). Naive Verhaltenstheorie. Stuttgart: Klett.

LeBoterf, G. (1994). De la compétence, essai sur un attracteur étrange. Paris: Les Editions d'Organisation.

LeBoterf, G. (1999). L'ingénierie des compétences. Paris: Les Editions d'Organisation.

Lepresle, C. (2003). Le projet professionnel en formation: une injonction paradoxale. Education permanente, 2, (155), $139-145$.

Lipari, D. (1995). Progettazione e valutazione nei processi formativi. Roma: Edizioni Lavoro.

Merlini, F. (2004). La comunicazione interrotta. Bari: Dedalo.

Meyrowitz, J. (1993). Oltre il senso di luogo. Bologna: Baskerville.

Où en est l'ingénierie de la formation? (2003) Education Permanente, 4, (152) [Numéro spécial].

Pain, A. (2003). L’ingénierie de la formation: état des lieux. Paris: L' Harmattan.

Perret-Clermont, A.-N. \& Zittoun, T. (2002). Esquisse d'une psychologie de la transition. Education permanente. Revue Suisse pour la Formation Continue, 12-15.

Reggiani, M. (2000). Coaching, Mentoring e dintorni: I mille percorsi dell'apprendimento. In P. L. Amietta (Ed.), I luoghi dell'apprendimento. Milano: F. Angeli.

Schön, D. (1983). The reflective practitioner. NY: Basic Books.

Schürch, D. (2001). Dove collocare la dimensione soggettiva del formatore? I quaderni dell'Istituto, $12-40$. Lugano: USI.

Selvatici, A. \& Grazia, D.A.M. (2004). Il Bilancio di competenze. Milano: F. Angeli.

Sennett, R. (1999). L’uomo flessibile. Le conseguenze del nuovo capitalismo sulla vita personale. Milano: Feltrinelli.

Suchman, L. (1987). Plans and situated action. Cambridge: Cambridge University Press.

Parole chiave: identità del formatore, ruoli del formatore, strutture modulari, flessibilità, attribuzione di senso, organizzazione del sapere. 


\section{Die Rollen des Ausbilders zwischen Tradition und Suche nach einer neuen Identität}

\section{Zusammenfassung}

Die Modularisierung der Bildungswege ist eine Antwort auf die verstärkte Nachfrage nach Flexibilisierung und Individualisierung der Ausbildungsgänge. Obschon diese Prozesse über bemerkenswerte Emanzipationspotenziale verfügen, können sie zu Unsicherheits- und Abhängigkeitsgefühlen führen. Durch die Einführung von geeigneten Massnahmen soll diesen Empfindungen so entgegenwirkt werden, dass der Heterogenität der Bildungswege, die aus mehr oder weniger voneinander unabhängigen Einheiten bestehen und jeweils unterschiedliche innere Logiken innehaben -wieder gerecht werden kann.

Gestützt auf die gesammelten Erfahrungen der Tätigkeiten des Schweizerischen Instituts für Berufspädagogik (SIBP) - die auf Aufgaben fokussieren, welche Fachpersonen im Ausbildungsbereich aufgrund der neuen Rahmenbedingungen wahrnehmen müssen - schlagen wir drei Rollen des Ausbildners vor: die Rolle des Managers, der Lehrkraft und des Begleiters. Kommen Ersterem insbesondere Aufgaben im Zusammenhang mit der Struktur der Bildungswege und deren Verwaltung zu, ist die Lehrkraft - deren Tätigkeit normalerweise im klar definierten Rahmen eines Moduls steht - für die Restrukturierung des Wissens (vermehrt von der Disziplin als natürliche Referenz entkoppelt) anhand neuer Ordnungskriterien wie etwa das Kompetenzprofil, verantwortlich. Eine zunehmende Bedeutung erhält die Rolle des Begleiters durchBeziehungsaspekte (Referenzpunkte für die Auszubildenden) und durch die Unterstützung bei den Lernprozessen (Metakognition, Anwendung diversifizierter Bildungsinstrumente). Die Bildungsaufgaben scheinen sich so vermehrt auf methodologische und beziehungsspezifische Aspekte zu konzentrieren, wobei die Sinngebungsprozesse ein verstärktes Gewicht erhalten, die solche innerhalb modularer Strukturen nicht mehr als selbstverständlich vorausgesetzt werden können.

Die Funktionen, welche die drei erarbeiteten Profile charakterisieren, können in diversen Kontexten und in Abhängigkeit zu diesen - besonders im Hinblick auf einen modularen Bildungsweg - in unterschiedlichen Kombination gefunden werden. Daraus ergibt sich eine Reihe von Fragestellungen, die die Präsentation und Konstruktion von professioneller Identität betreffen: Falls verstärkt eine Aufteilung der Arbeitstätigkeit stattfindet, die mit einer zunehmenden Spezialisierung der drei Berufsrollen in den eigenen Kompetenzbereichen einhergeht, welche Erwartungen etwa in der Präsentation werden dann bei den Lernenden hervorgerufen? Wie gestaltet sich die persönliche Wahrnehmung der Professionellen hinsichtlich der eigenen Identität?

Schlagworte: Professionelle Identität, Professionelle Rollen, modulare Strukturen, Flexibilität, Wissensorgnaisation, Bedeutungszuweisung 


\section{Les rôles du formateur entre tradition et construction d'une nouvelle identité.}

\section{Résumé}

La modularisation constitue une réponse des systèmes de formation à la demande croissante de flexibilité et d'individualisation des parcours d'apprentissage. Si d'un côté ces processus sont porteurs d'un potentiel d'émancipation remarquable, de l'autre ils peuvent engendrer des sentiments d'insécurité et de dépendance qui appellent à des mesures aptes à faire face à l'hétérogénéité propre à des parcours structurés en unités plus au moins indépendantes entre elles et donc porteuses de logiques à chaque fois différentes.

A partir des expériences conduites à l'ISPFP, notre réflexion prend en considération les tâches que les formateurs sont appelés à assumer dans ces nouveaux contextes de formation et elle nous amène à identifier trois rôles: le manager, l'enseignant et l'accompagnateur. Si l'activité du premier s'explicite surtout par rapport à la structure des parcours et à leur gestion, on demande à l'enseignant dont le rôle se joue normalement dans le cadre d'un module - de restructurer la connaissance (qui ne trouve plus son référant naturel dans la discipline) en fonction de nouveaux critères d'ordre, par exemple le profil de compétence visé. Le rôle d'accompagnement assume une importance croissante par rapport aux aspects qui touchent à la relation (point de référence pour les apprenants) et aux processus d'apprentissage (métacognition, emploi d'instruments différenciés). Les tâches de la formation semblent donc se concentrer sur les aspects méthodologiques et relationnels avec une attention particulière aux processus d'attribution de sens qui, dans le contexte de structures modulaires, ne peuvent plus être considérés comme acquis à priori.

Les fonctions qui caractérisent ces trois profils de formateur peuvent se combiner de façons différentes par rapport aux différentes situations et en particulier par rapport à la typologie de parcours modulaire. On se trouve donc confrontés à une série d'interrogations qui touchent à l'identité du formateur: si la tendance est à une division du travail qui voit les trois rôles professionnels toujours plus spécialisés dans les secteurs de compétence respectifs, quelles sont les représentations et les attentes induites chez les apprenants? Quelle perception le formateur peut-il avoir de lui même par rapport à son identité en tant que professionnel?

Mots clés: Identité du formateur, rôles du formateur, structures modulaires, flexibilité, construction de sens, organisation des savoirs. 


\section{The role of the trainer between tradition and search for a new identity.}

\section{Summary}

Module training is an answer to the increasing demand of flexibility and individualization of vocational training. On one hand these two trends arise a considerable potential of emancipation, but on the other one they might cause insecurity and dependence, so that the introduction of measures able to recompose the heterogeneity in trainings composed by different units - more or less independent - and bearings of different internal logics becomes necessary. Following our thoughts and experiences at the ISPFP, the educator belonging to the module training format must adopt three roles: the manager, the teacher and the "scaffolder". The first one has duties connected with the structure of the training and management; the teacher, whose duties normally unfold in a well-defined module, must rebuild the knowledge - not referring to the discipline anymore following new criteria, for example the outline of an aimed competence. Then, scaffolding gains more and more importance in the relationship aspects (a reference point for the person in the formation process) and supporting the learning procedures (metacognition, use of different formation instruments). Thus, the module structure formation concentrates itself on its methodological and relationship aspects with a specific focus on competence procedures, which can't be given for granted anymore.

The duties, which characterize the three roles previously seen, can combine themselves in a different way according to the contingent situations and more specifically to the type of module training. This peculiarity of module training faces us up to a series of questions relating to the educator's identity: if the emerging tendency is the subdivision of the work, requiring the three professional roles becoming more and more specialized in their respective competence sectors, what will be the induced representations and the expectations of the people in the process of formation? What will be the educator's perception of his own professional identity?

Key words: Trainer identity, trainer roles, module training, sense making, knowledge building. 
T $h$ e $\mathrm{m} \mathrm{a}$ 